\title{
-NOTES-
}

\section{AN ELECTRICAL NETWORK WITH VARYING PARAMETERS*}

\author{
By C. P. GADSDEN (Tulane University)
}

The aim of this paper is to study the free vibrations in a simple linear system whose parameters may vary with time.

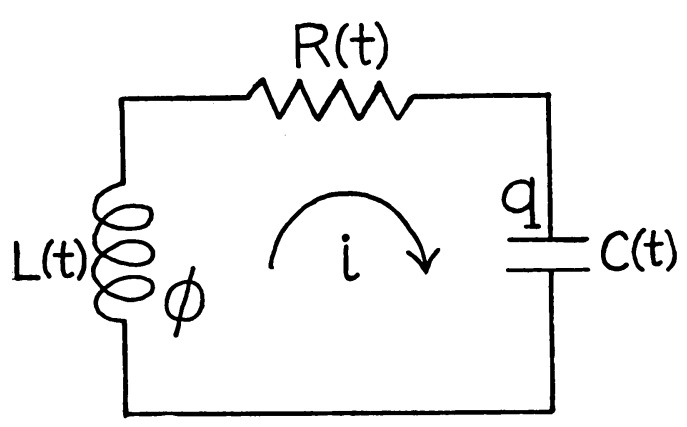

FIG. 1

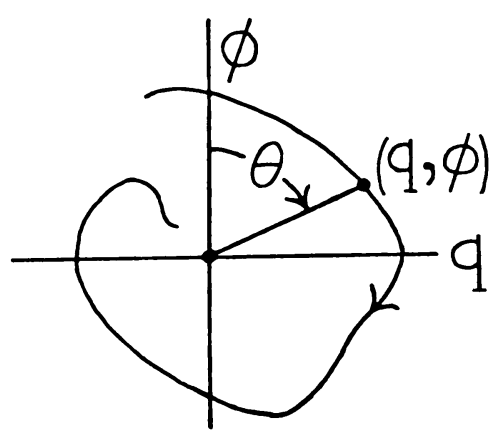

Fig. 2

The system we will consider is the electrical circuit of Fig. 1. The fundamental relations are

$$
\begin{gathered}
\Phi=L i=L \frac{d q}{d t}, \\
\frac{d \Phi}{d t}+R i+C^{-1} q=0,
\end{gathered}
$$

where $\Phi$ is the flux linkages, $q$ the condenser charge, and $i$ the current. ${ }^{1}$ The energy stored in the system is $E=\frac{1}{2} C^{-1} q^{2}+\frac{1}{2} L^{-1} \Phi^{2}$, and its rate of change is found to be, using (1) and (2),

$$
\frac{d E}{d t}=-R i^{2}-\frac{1}{2} C^{-2} q^{2} \frac{d C}{d t}-\frac{1}{2} L^{-2} \Phi^{2} \frac{d L}{d t} .
$$

From (3) it is clear that if $C$ or $L$ is varied, energy is added to or extracted from the circuit. This fact has been explored by Barrow, ${ }^{2}$ Erdelyi, ${ }^{3}$ Minorsky $^{4}$ and others to

*Received Sept. 19, 1949. Revision received Dec. 28, 1949. This investigation was carried out under a contract with the Office of Naval Research.

${ }^{1}$ Note that the self-induced voltage is taken to be $d(L i) / d t$, not $L d i / d t$. The former assumption was made by L. A. Pipes, Operational and matrix methods in linear variable networks, Phil. Mag. 25, .585-600 (1938). The latter was made by Barrow (Ref. 2, p. 212) and by Minorsky (Ref. 4), and is incorrect.

${ }^{2}$ W. L. Barrow, On the oscillations of a circuit having a periodically varying capacitance, Proc. I. R. E. 22, 201-212 (1934).

${ }^{3} \mathrm{~A}$. Erdelyi, Ueber die freien Schwingungen in Kondensatorkreisen mit periodisch veraenderlicher Kapazitaet, Ann. Physik (5) 19, 585-622 (1934).

${ }^{4} \mathrm{~N}$. Minorsky, On parametric excitation, J. Franklin Inst. 240, 25-46 (1945). See bibliography. 
show that the vibrations may be maintained, or even built up, in spite of the dissipation $R i^{2}$, by giving $C$ or $L$ a suitable periodic variation.

It turns out to be convenient to discuss the behavior of the circuit in terms of the quantities $q$ and $\Phi$ together. To this end we eliminate $i$ from (1) and (2) and consider the system of equations

$$
\begin{aligned}
& \frac{d q}{d t}=L^{-1} \Phi, \\
& \frac{d \Phi}{d t}=-C^{-1} q-R L^{-1} \Phi .
\end{aligned}
$$

It is assumed that $L(t), C(t)$ and $R(t)$ are continuous functions of $t$, with continuous derivatives, whose values remain between some positive bounds: $0<m<L(t), C(t)$, $R(t)<M$. Under these assumptions the coefficients $L^{-1},-C^{-1}$ and $-R L^{-1}$ in (4) are continuous; this is sufficient to assure that any pair $q\left(t_{0}\right), \Phi\left(t_{0}\right)$ of initial values uniquely determines a solution $q(t), \Phi(t)$ of (4); in particular, if $q\left(t_{0}\right)=\Phi\left(t_{0}\right)=0$, we have the trivial solution $q(t) \equiv \Phi(t) \equiv 0$.

Let us picture a non-trivial solution of (4) in the $q$, $\Phi$ plane (Fig. 2). From the preceding remark the solution curve never passes through the origin. The shape of the curve depicted can be justified as follows. From (4) we obtain $d \Phi / d q=-L C^{-1}(q / \Phi)-R$. Thus the slope of the curve is negative in the first and third quadrants. ${ }^{5} \mathrm{Next}$, consider the polar angle $\theta$, which we will think of as changing continuously, not as reduced modulo $2 \pi$. To be precise we define the function $\theta=\theta(t)$ to be the unique continuous function of $t$ satisfying the relations $\Phi=\rho \cos \theta, q=\rho \sin \theta$, and $0 \leq \theta\left(t_{0}\right)<2 \pi$, where $\rho=$ $\left(q^{2}+\Phi^{2}\right)^{1 / 2}>0$. For the rate of change of $\theta$ we have the expression

$$
\frac{d \theta}{d t}=L^{-1} \cos ^{2} \theta+R L^{-1} \sin \theta \cos \theta+C^{-1} \sin ^{2} \theta .
$$

In the first and third quadrants $\sin \theta \cos \theta \geq 0$ so that $d \theta / d t>M^{-1} \cos ^{2} \theta+M^{-1} \sin ^{2}$ $\theta=M^{-1}>0$. Hence the direction of increasing time is as shown by the arrow. This inequality shows also that the point $q, \Phi$ cannot remain continually in the first or third quadrant longer than a time $\frac{1}{2} M \pi$.

1. Oscillation. Two distinct types of behaviour are possible for a solution curve as $t \rightarrow \infty$ : (i) it may wind continually around the origin, so that $q(t)$ and $\Phi(t)$ have an oscillatory character (about the value zero), or (ii) after a certain time it may remain in the second or fourth quadrant, so that $q(t)$ and $\Phi(t)$ become non-oscillatory. With regard to these two possibilities we establish several properties.

I. Whether the vibrations are of the oscillatory or of the non-oscillatory type is independent of the initial values of charge and fux (provided not both are zero). This follows from the fact ${ }^{6}$ that the zeros of corresponding components of any two linearly independent solutions of (4) separate one another, while such zeros of non-trivial linearly dependent solutions coincide. Thus either all such solutions are oscillatory or else all are non-oscillatory.

${ }^{5}$ Note that the slope at the point $q$, $\Phi$ depends, in general, upon $t$; thus the diagram of Fig. 2 differs somewhat from the usual "phase-plane" diagram.

${ }^{6} \mathrm{M}$. Bocher, On the real solutions of systems of two homogeneous linear differential equations of the first order, Trans. Amer. Math. Soc. 3, 196-215 (1902). 
We give now an estimate of the number of complete oscillations of $q(t)$ or $\Phi(t)$ that occur in an arbitrary time interval $\left(t_{1}, t_{2}\right)$. This number can be measured by the quantity $(2 \pi)^{-1}\left[\theta\left(t_{2}\right)-\theta\left(t_{1}\right)\right]$. However, instead of $\theta$ we use the angle $\psi$ defined by $\psi=\operatorname{arc}$ tan $(r q / \Phi)$ and the requirement that $|\psi-\theta|<\frac{1}{2} \pi$, where $r=r(t)$ is any positive function of $t$ with a continuous derivative. It is clear that $\theta$ and $\psi$ always lie in the same quadrant, so that the quantity $(2 \pi)^{-1}\left[\psi\left(t_{2}\right)-\psi\left(t_{1}\right)\right]$ will also serve our purpose. Similar to (5) we have the expression

$$
\frac{d \psi}{d t}=r L^{-1} \cos ^{2} \psi+\left(R L^{-1}+r^{-1} \frac{d r}{d t}\right) \sin \psi \cos \psi+r^{-1} C^{-1} \sin ^{2} \psi
$$

or in terms of $2 \psi$,

$$
\frac{d 2 \psi}{d t}=r L^{-1}+r^{-1} C^{-1}+\left(r L^{-1}-r^{-1} C^{-1}\right) \cos 2 \psi+\left(R L^{-1}+r^{-1} \frac{d r}{d t}\right) \sin 2 \psi .
$$

From the last relation we have the inequalities

$$
A(t) \leq \frac{d \psi}{d t} \leq B(t)
$$

where

$$
A(t), B(t)=\frac{1}{2}\left\{r L^{-1}+r^{-1} C^{-1} \mp\left[\left(r L^{-1}-r^{-1} C^{-1}\right)^{2}+\left(R L^{-1}+r^{-1} \frac{d r}{d t}\right)^{2}\right]^{1 / 2}\right\} .
$$

Integrating (6) between $t_{1}$ and $t_{2}$ we have

$$
2 \pi a \leq \psi\left(t_{2}\right)-\psi\left(t_{1}\right) \leq 2 \pi b,
$$

where

$$
a=(2 \pi)^{-1} \int_{t_{1}}^{t_{2}} A(t) d t, \quad b=(2 \pi)^{-1} \int_{t_{1}}^{t_{2}} B(t) d t
$$

Hence:

II. The number of complete oscillations of $q(t)$ and $\Phi(t)$ between the times $t_{1}$ and $t_{2}$ is at least $a$ but does not exceed $b$. Also, if $\lim a=\infty$ as $t_{2} \rightarrow \infty$, the vibrations are oscillatory, while if $\lim b<\infty$ as $t_{2} \rightarrow \infty$ they are non-oscillatory.

In using the preceding result the function $r(t)$ should be chosen to give the best estimate. This choice can affect the values of $a$ and $b$ considerably. For example, if $L=1, C=1 / 4, R=0$, the choice $r=1$ gives $1 \leq d \psi_{1} / d t \leq 4$, whereas if we take $r=$ $L^{1 / 2} C^{-1 / 2}=2$ we have $d \psi_{2} / d t=2$, for which the error is zero. The question of determining in general the best $r(t)$ leads to a problem in the calculus of variations; however, the Euler equation seems to be unmanageable.

We can of course get oscillation and non-oscillation criteria by reducing the system (4) to a single second order equation of the form

$$
\frac{d^{2} y}{d s^{2}}+f(s) y=0
$$

in such a way that $y(s)$ is oscillatory or non-oscillatory if and only if $q(t)$ or $\Phi(t)$ is likewise. This can be done in several ways. When it has been accomplished the classical 
comparison theorems of Sturm and other oscillation conditions ${ }^{\mathbf{7 . 8}}$ for (7) can be applied. The following result illustrates the procedure.

III. If $F(t)=L C^{-1}-(R / 2)^{2}-(L / 2) d R / d t \geq k>0$ for $t>T$, where $k$ is a constant, the vibrations are oscillatory, while if $F(t) \leq 0$ for $t>T$ they are non-oscillatory for $t>T$. To show this first eliminate $\Phi$ from (4) and obtain

$$
\frac{d}{d t}\left(L \frac{d q}{d t}\right)+R \frac{d q}{d t}+C^{-1} q=0
$$

Next introduce a new independent variable $s$ defined by $s=\int_{T}^{t} L^{-1} d t$. Since $d s / d t>0$, $t$ is also a single-valued function of $s$, say $t=g(s)$. Also, $s>M^{-1}(t-T)$, so that $s \rightarrow \infty$ as $t \rightarrow \infty$. Putting $q(t)=q[g(s)]=q_{1}(s)$, and similarly $R(t)=R_{1}(s), L(t)=L_{1}(s)$, $C(t)=C_{1}(s),(8)$ becomes

Finally in (9) put

$$
\frac{d^{2} q_{1}}{d s^{2}}+R_{1} \frac{d q_{1}}{d s}+L_{1} C_{1}^{-1} q_{1}=0
$$

$$
q_{1}(s)=y(s) \exp \int_{0}^{s}-\frac{1}{2} R_{1}(s) d s
$$

and get an equation of the form (7) where

$$
f(s)=L_{1} C_{1}^{-1}-\left(\frac{1}{2} R_{1}\right)^{2}-\frac{1}{2} d R_{1} / d s=L C^{-1}-\left(\frac{1}{2} R\right)^{2}-\left(\frac{1}{2} L\right) d R / d t=F(t) .
$$

A comparison theorem ${ }^{9}$ of Sturm applied to (7) shows that $y(s)$ is non-oscillatory as $s \rightarrow \infty$ if $f(s) \leq 0$ for $s>0$, that is, if $F(t) \leq 0, t>T$. But if $y(s)$ is non-oscillatory, (10) shows that $q_{1}(s)=q(t)$ is also. On the other hand, if $f(s) \geq k>0$ this theorem shows that $y(s)$ oscillates at least as rapidly as the solutions of $(7)$ with $f(s)=k$. But if $y(s)$ is oscillatory, so is $q(t)$.

IV. If the vibrations are non-oscillatory, they are also transient, that is, $\lim q(t)=$ $\lim \Phi(t)=0$ as $t \rightarrow \infty$. To prove this note first that if the vibrations are non-oscillatory, $q(t)$ and $\Phi(t)$ have constant and opposite signs after some time $T$, inasmuch as $q, \Phi$ cannot remain indefinitely in the first or third quadrants of Fig. 2. Thus for $t>T$ the functions $|q(t)|$ and $|\Phi(t)|$ satisfy the system

$$
\begin{aligned}
& \frac{d|q|}{d t}=-L^{-1}|\Phi|, \\
& \frac{d|\Phi|}{d t}=C^{-1}|q|-R L^{-1}|\Phi| .
\end{aligned}
$$

From (11a) $|q(t)|$ decreases monotonically as $t$ increases and so approaches a definite limit $|q(\infty)|$ as $t \rightarrow \infty$. From (11b) we have

$$
\frac{d}{d t}\left[|\Phi| \exp \int_{T}^{t} R L^{-1} d t\right]=C^{-1}|q| \exp \int_{T}^{t} R L^{-1} d t .
$$

${ }^{7} \mathrm{R}$. Bellman, $A$ survey of the theory of boundedness, stability and asymptotic behavior of solutions of linear and non-linear differential and difference equations, Office of Naval Research, Department of the Navy, Washington, D. C., 1949, p. 116-136.

${ }^{8}$ A. Wintner, A criterion of oscillatory stability, Q. Appl. Math. 7, 115-117 (1949).

${ }^{\circ} \mathrm{E} . \mathrm{L}$. Ince, Ordinary differential equations, Dover Publications, New York, p. 226. 
Integrating (12) between $T$ and $t$ gives

$$
|\Phi(t)|=\left[|\Phi(T)|+\int_{T}^{t} C^{-1}|q|\left(\exp \int_{T}^{t} R L^{-1} d t\right) d t\right]\left(\exp \int_{T}^{t} R L^{-1} d t\right)^{-1} .
$$

To the right side of (13) we apply an extension ${ }^{10}$ of L'Hospital's rule concerning the limits of the quotient $f(t) g^{-1}(t)$ as $t \rightarrow \infty$ : if $\lim |g(t)|=\infty$ as $t \rightarrow \infty$, and $f(t)$ and $g(t)$ are never simultaneously zero or infinite in the interval $(T, \infty)$ then

$$
\limsup _{t \rightarrow \infty} \frac{f(t)}{g(t)} \leq \limsup _{t \rightarrow \infty} \frac{f^{\prime}(t)}{g^{\prime}(t)}
$$

and

$$
\liminf _{t \rightarrow \infty} \frac{f(t)}{g(t)} \geq \liminf _{t \rightarrow \infty} \frac{f^{\prime}(t)}{g^{\prime}(t)},
$$

where the primes denote derivatives. In our case

$$
|g(t)|=\exp \int_{T}^{t} R L^{-1} d t \geq \exp m M^{-1}(t-T) \rightarrow \infty \quad \text { as } \quad t \rightarrow \infty .
$$

Hence we have

$$
\begin{aligned}
\lim \sup |\Phi(t)| & \leq \lim \sup \frac{C^{-1}|q(t)|}{R L^{-1}} \leq \lim \sup m^{-2} M|q(t)| \\
& =|q(\infty)| m^{-2} M<\infty,
\end{aligned}
$$

and

$$
\begin{aligned}
\infty>\lim \inf |\Phi(t)| & \geq \liminf \frac{C^{-1}|q(t)|}{R L^{-1}} \geq \lim \inf M^{-2} m|q(t)| \\
& =|q(\infty)| M^{-2} m \geq 0,
\end{aligned}
$$

where all limits are taken as $t \rightarrow \infty$. Now suppose $\lim \inf |\Phi(t)|>0$. Then from the definition of the lim inf, if $\epsilon$ is a number such that $0<\epsilon<\lim \inf |\Phi(t)|$, there is a $t_{1}$ such that for $t>t_{1},|\Phi(t)|>\epsilon$. Hence from (11a) $d|q| / d t<-M^{-1} \epsilon$. Integration of this inequality gives $0<|q(t)| \leq\left|q\left(t_{1}\right)\right|-M^{-1} \epsilon\left(t-t_{1}\right)$. But as $t \rightarrow \infty$ the right side of this inequality $\rightarrow-\infty$. This contradiction shows that $\lim \inf |\Phi(t)|=0$. Then from (15), $|q(\infty)|=0$ and from (14), $\lim \sup |\Phi(t)|=0$, so that $\lim q(t)=\lim \Phi(t)=0$ as $t \rightarrow \infty$.

2. Stability. In this section we prove some facts concerning the stability of the solutions $q(t), \Phi(t)$ of (4). It should be emphasized that unstable solutions are indeed possible. This has been shown both theoretically and experimentally. ${ }^{2}$

We will say that a function $h(t)$ is stable if it remains bounded as $t \rightarrow \infty$, i.e., if lim sup $|h(t)|<\infty$. Our first result shows that $q(t)$ and $\Phi(t)$ have the same stability behavior. The proof requires the assumption that $d R / d t$ remains bounded as $t \rightarrow \infty$.

${ }^{10} \mathrm{~L}$. M. Graves, The theory of functions of real variables, McGraw-Hill Book Co., Inc., New York, 1946, p. 83. 
V. If $q(t)$ is stable or transient, $\Phi(t)$ is likewise, and conversely. From (4) we get an equation like (12)

$$
\frac{d}{d t}\left[\Phi \exp \int_{T}^{t} R L^{-1} d t\right]=-C^{-1} q \exp \int_{T}^{t} R L^{-1} d t
$$

and like (13)

$$
\Phi(t)=\left[\Phi(T)-\int_{T}^{t} C^{-1} q\left(\exp \int_{T}^{t} R L^{-1} d t\right) d t\right]\left(\exp \int_{T}^{t} R L^{-1} d t\right)^{-1} .
$$

Hence

$$
|\Phi(t)| \leq\left[|\Phi(T)|+\int_{T}^{t} C^{-1}|q|\left(\exp \int_{T}^{t} R L^{-1} d t\right) d t\right]\left(\exp \int_{T}^{t} R L^{-1} d t\right)^{-1} .
$$

Applying the extended L'Hospital rule to (16) gives

$$
\lim \sup |\Phi(t)| \leq \lim \sup \frac{C^{-1}|q(t)|}{R L^{-1}} \leq \frac{M}{m^{2}} \lim \sup |q(t)| .
$$

Thus if $q(t)$ is stable, so is $\Phi(t)$, while if $q(t) \rightarrow 0$ as $t \rightarrow \infty$, so does $\Phi(t)$. To show the converse we obtain from (4)

$$
\frac{d q}{d t}+R^{-1} C^{-1} q=-R^{-1} \frac{d \Phi}{d t} .
$$

This equation is satisfied by

$$
q(t)=\left[q(T)-\int_{T}^{t} R^{-1} \frac{d \Phi}{d t}\left(\exp \int_{T}^{t} R^{-1} C^{-1} d t\right) d t\right]\left(\exp \int_{T}^{t} R^{-1} C^{-1} d t\right)^{-1} .
$$

An integration by parts puts (17) in the form

$$
\begin{aligned}
q(t)=- & R^{-1} \Phi(t)+[q(T) \\
& \left.+\int_{T}^{t} \Phi R^{-2}\left(C^{-1}-\frac{d R}{d t}\right)\left(\exp \int_{T}^{t} R^{-1} C^{-1} d t\right) d t\right]\left(\exp \int_{T}^{t} R^{-1} C^{-1} d t\right)^{-1} .
\end{aligned}
$$

Let $k$ be an upper bound for $R^{-2}\left|C^{-1}-d R / d t\right|$ in the interval $t>T$. Then

$$
\begin{aligned}
|q(t)| \leq R^{-1}|\Phi(t)|+[|q(T)| \\
\left.\quad+k \int_{T}^{t}|\Phi|\left(\exp \int_{T}^{t} R^{-1} C^{-1} d t\right) d t\right]\left(\exp \int_{T}^{t} R^{-1} C^{-1} d t\right)^{-1} .
\end{aligned}
$$

Hence lim sup $|q(t)| \leq\left(m^{-1}+k M^{2}\right)$ lim sup $|\Phi(t)|$. Thus if $\Phi(t)$ is stable or transient, $q(t)$ is likewise.

Next let us see if we have an analog for stability of the result $I$ for oscillation. That is, does the behavior of the circuit with regard to stability depend upon the initial conditions? Strictly speaking it does. Thus one (non-trivial) solution of the system (4) may be stable and another unstable. In such a case, however, we can see that the system 
is essentially unstable. That is, the possible initial values $q_{0}, \Phi_{0}$ which give rise to stable solutions all lie on a certain straight line through the origin of the $q, \Phi$ plane. For suppose $q_{1}(t), \Phi_{1}(t)$ is a (non-trivial) stable solution and $q_{2}(t), \Phi_{2}(t)$ an unstable solution. These are clearly linearly independent, so that every solution of (4) has the form

$$
\begin{gathered}
q(t)=a q_{1}(t)+b q_{2}(t), \\
\Phi(t)=a \Phi_{1}(t)+b \Phi_{2}(t),
\end{gathered}
$$

where $a$ and $b$ are constants. If the solution (18) is to be stable we must certainly have $b=0$. Putting $t=t_{0}$ in (18) and eliminating $a$ results in

$$
q_{1}\left(t_{0}\right) \Phi_{0}-\Phi_{1}\left(t_{0}\right) q_{0}=0,
$$

a straight line in $q_{0}, \Phi_{0}$.

As stability criteria we have, besides IV,

VI. All solutions of (4) are stable if either

$$
\frac{d}{d t}\left(\frac{C}{L}\right) \leq 2 R C L^{-2}
$$

or

$$
\frac{d}{d t}\left(\frac{C}{L}\right) \geq 0
$$

holds for $t>T$ for some $T$. To prove that (i) is sufficient to insure stability consider the positive form $E_{1}=C L^{-1} \Phi^{2}+q^{2}$. If $q, \Phi$ is a solution of (4) we have

$$
\frac{d E_{1}}{d t}=-\left[2 R C L^{-2}-\frac{d}{d t}\left(\frac{C}{L}\right)\right] \Phi^{2},
$$

so that $E_{1}$ is a non-increasing function of $t$ for $t>T$ if (i) holds. Being positive, $E_{1}$ is therefore bounded as $t \rightarrow \infty$. Finally $E_{1}>m M^{-1} \Phi^{2}+q^{2}>0$, so that $q(t)$ and $\Phi(t)$ are stable. To prove condition (ii) consider $E_{2}=\Phi^{2}+L C^{-1} q^{2}$. In this case we have

$$
\frac{d E_{2}}{d t}=-\left[L^{2} C^{-2} \frac{d}{d t}\left(\frac{C}{L}\right)\right] q^{2}-2 R L^{-1} \Phi^{2} .
$$

Thus if (ii) holds, $E_{2}$ is non-increasing and $q(t)$ and $\Phi(t)$ are stable. The conditions (i) and (ii) show that the derivative of $C / L$ must vary continually outside both the limits 0 and $2 R C L^{-2}$ if the vibrations are unstable.

3. Remarks. The above results apply at once of course to the dual of the circuit of Fig. 1, namely a parallel connection of one of each of the three types of elements, as well as to mechanical or acoustical analogs of these circuits. The question of the description of the forced vibrations may be raised. As is well known from the theory of systems of linear differential equations, the forced component can be expressed in terms of the free vibrations. Thus for any discussion of the forced vibrations it should be helpful to know something of the character of the (relatively) simpler free vibrations. 